\title{
FORMAS TERMINALES DE HIPPIDION (MAMMALIA, PERISSODACTYLA) DE LOS YACIMIENTOS DEL PLEISTOCENO TARDIO-HOLOCENO DE LA PATAGONIA (ARGENTINA Y CHILE) ${ }^{(1)}$
}

\author{
M.T. Alberdi $(*)$, A.N. Menegaz $\left({ }^{* *}\right)$ y J.L. Prado (**)
}

\begin{abstract}
RESUMEN
El presente estudio comprende una revisión de las formas terminales de caballos sudamericanos recuperados de sitios arqueológicos de la Patagonia. Se comparan con las formas del noroeste argentino. Incluye aspectos paleoambientales y cronológicos relacionados.
\end{abstract}

Palabras clave: Perissodactyla, Hippidion, Limite Pelistoceno-Holoceno, Argentina.

\begin{abstract}
The present study is a revision of the terminal forms of the South-American horses coming from the archeological sites of Patagonia. They are compared with those from the Northwest of Argentine. The study also includes paleoenvironmental and cronological related aspects.
\end{abstract}

Key words: Perissodactyla, Hippidion, Pleistocene-Holocene boundary, Argentina.

\section{Introducción}

La región Patagónica presenta la particularidad, desde el punto de vista arqueológico, de poseer sitios de edad temprana dentro de la secuencia sudamericana (Bryan, 1986).

Estos sitios tempranos - 13.000 a 8.500 B.P.(Mengoni Gon̄alons, 1986; Ortiz Troncoso, 1981) han sido, en algunos casos, trabajados desde el siglo pasado (Hauthal et al., 1899; Nordenskjöld, 1900), aportando interesantes y numerosos restos faunísticos (Mengoni Goñalons, 1983, 1986).

Entre tales conjuntos faunísticos se encuentran generalmente restos de caballos americanos, los cuales si bien fueron tratados en cada sitio en particular (desde un enfoque arqueológico), hasta el momento no han sido motivo de un estudio específico que los relacione entre sí. Esta temática se ve reflejada en la multiplicidad de denominaciones taxonómicas con los que han sido referidas en la bibliografía especializada (Roth, 1899; San- guinetti de Bormida, 1976; Cardich, 1977; Cardich et al., 1973; Mengoni Goñalons, 1976-80; Bird, 1938; Poulain-Joisein, 1963).

El presente estudio comprende una revisión de los materiales de caballos fósiles americanos recuperados de sitios arqueológicos de Patagonia y su relación con materiales de otras regiones. Asimismo se contemplarán aspectos paleoambientales y cronológicos relacionados.

\section{Características de la región}

La Patagonia constituye la región más austral del continente americano. Abarca el territorio comprendido al S de los 39" de latitud sur, hasta el archipiélago Fueguino inclusive (República Argentina y Chile).

Se pueden reconocer dos grandes subregiones desde el punto de vista fisiográfico (García Castellanos, 1984):

1) El área cordillera, que incluye las unidades estructurales de Cordillera Neuquina, Cordillera Patagónica austral y Cordillera Fueguina.

(1) Este trabajo se ha realizado dentro del Convenio bilateral CONICET-CSIC, «Correlaciones del Cuaternario reciente entre Sudamérica y Europa con énfasis especial en las faunas españolas y argentinas».

(*) Museo Nacional de Ciencias Naturales. CSIC. José Gutiérrez Abascal, 2. 28006 Madrid (España).

(**) CIC. Museo de La Plata. Paseo del Bosque, s/n. La Plata 1900 (Argentina). 
2) El área de la Meseta Patagónica, que incluye a los Macizos del Deseado y Somuncurá y a la Patagonia extrandina.

La zona cordillera posee montañas relativamente bajas. Las altitudes máximas decrecen desde, aproximadamente $3.500 \mathrm{~m}$ al $\mathrm{N}$, hasta $1.000 \mathrm{~m}$ en la Isla Grande de Tierra del Fuego.

La diferencia gradual de altitudes hacia el $\mathrm{S}$ condiciona diferencialmente el paso de la humedad de los vientos del Pacífico. El clima, en consecuencia, varía de frío y seco al $\mathrm{N}$ hasta muy frío $\mathrm{y}$ húmedo al $\mathrm{S}$ del lado argentino. En la vertiente chilena las condiciones de humedad son constantes.

Por otra parte, el clima de la meseta patagónica es subdesértico, marcadamente continental.

En la región se registró durante el Pleistoceno final un avance parcial del hielo (Auer, 1956, 1970; Richardi y Rolleri, 1980). Por evidencias palinológicas, este momento se vincularía al retiro de los hielos y postglacial temprano (Heuser, 1966, 1974; Mercer, 1968, 1976 a y b), el cual comenzó hacia el 10.000 BP manteniéndose la situación interglacial hasta, aproximadamente, el 6.000 BP (Mercer, 1968, 1976 a y b). Consecuentemente la extinción de ésta y otras formas pleistocénicas podrían estar relacionadas con el deterioro climático (Tambussi y Tonni, 1985). Sin embargo, el comportamiento climático durante el postglacial no habría sido homogéneo para toda la región patagónica, registrándose fluctuaciones en temperatura y humedad (Feruglio, 1950; Heuser, 1966, 1974; Paskoff, 1977).

\section{Materiales y métodos}

Los materiales considerados provienen de los siguientes sitios arqueológicos: Cueva del Mylodon, Cueva Fell, Cueva Las Buitreras, Cuevas de Los Toldos III y II. Cueva Pali Aike y Cerro Soto (fig. 1).

Los restos fósiles analizados se encuentran despositados en las colecciones de Paleontología y Arqueología del Museo de La Plata (MLP), Museo Etnográfico de Buenos Aires e Instituto de Ciencias Antropológicas de la Universidad de Buenos Aires.

Con respecto al análisis osteométrico se utilizó el método elaborado por Alberdi (1974) y trabajos posteriores para el género Hipparion, así como las recomendaciones y normas elaboradas en la "Hipparion Conference», celebrada en New York en 1981 (en prensa), basadas, en parte, en Eissenman (1979). Todas las dimensiones se expresan en milímetros.

Los detalles acerca de la cronología y estratigrafía de los sitios corresponden a citas textuales de los trabajos originales.

\section{Situación geográfica, estratigráfica y cronológica de los sitios con Hippidion (Figura 1)}

\section{Cueva del Mylodon (=Eberhardt= Ultima Esperanza)}

La cueva se encuentra situada próxima a la frontera argentino-chilena, en la latitud de Puerto Natale (Chile), a los $51^{\circ} 03^{\prime}$ '05" de latitud S y $72^{\circ}$ 36' de longitud W.

Este sitio fue excavado en 1899 por Hauthal, por Nordenskjöld en el mismo año, por Emparaire et al., 1963 y por Saxon en 1976.

Las excavaciones de Hauthal proporcionaron abundante material faunístico, estudiado por Roth $(1899,1902)$ y Lemanh Nistche (1899, 1902). Lamentablemente ninguno de estos trabajos considera las relaciones estratigráficas de los hallazgos, tratando el conjunto faunístico como una unidad. Roth (1899), en base a estos materiales, crea la especie Onohippidium saldiasi.

Por su parte, Nordenskjöld diferenció de arriba hacia abajo 3 capas: A, B y C. Las capas B y C contienen restos de caballos.

Dataciones radiocarbónicas del colágeno de una vértebra de Mylodon, posiblemente correspondiente a la base de la capa C (Mengoni, 1986), arrojaron una edad de $13.260 \pm 115$ (Lu. 794, Hakanson, 1976).

Posteriormente Saxon diferenció 12 niveles estratigráficos correlacionando parte del 5 y 6 con la capa B de Nordenskjöld, en tanto que del nivel 7 al 10 corresponderían al C.

El nivel 7, techo de la capa $C$ según Saxon (1976), ha sido datado reiteradamente a partir de la misma muestra de carbón:

\subsection{4 \pm 52 (Saxon, 1978: 4 BM 1204A) \\ 5.643 \pm 60 (Borrero, 1977: 84 BM 1204B) \\ 5.395 58 (Borrero, 1977: 84 BM 1201B) \\ 5.366 445 (Saxon, 1976: 66 y 71 BM 1202).}

Todas estas fechas son radiocarbónicamente idénticas, por lo tanto no hay una verdadera contrastación de las edades obtenidas. Por otra parte no existe en ninguno de los trabajos realizados, a partir de estas excavaciones, un análisis detallado de la correspondencia muestra-evento. Sin embargo, en la literatura arqueológica estos datos han sido utilizados para proponer la supervivencia de las especies pleistocénicas hasta el Holoceno medio, ya que la capa B de Nordenskjöld, con el caballo y el perezoso terrestre (ver cuadro 1), suprayace a la capa 7 de Saxon datada (ver Mengoni Goñalons, 1976-80; Borrero, 1977; Saxon, 1976, 1978).

\section{Los Toldos}

El yacimiento arqueológico de Los Toldos está situado en el paraje Cañadón de las cuevas de la Estancia Los Toldos, a los $47^{\circ} 22^{\prime}$ de latitud S y $68^{\circ} 58^{\prime}$ de longitud W (provincia de Santa Cruz).

El sitio fue descubierto por Aparicio y Frengüelli en 1933 (Aparicio, 1933). Más tarde fue estudiado por Menghin, quien realizó una serie de sondeos en las cuevas II y III, en 1951 y 1954 (Menghin, 1952, 1957, 1965).

\section{Sitio III de Los Toldos}

A los trabajos de Menghin (1952, 1957, 1965) continuaron los trabajos de Cardich (Cardich et al., 1973; Cardich, 1977; Cardich y Miotti, 1983). 


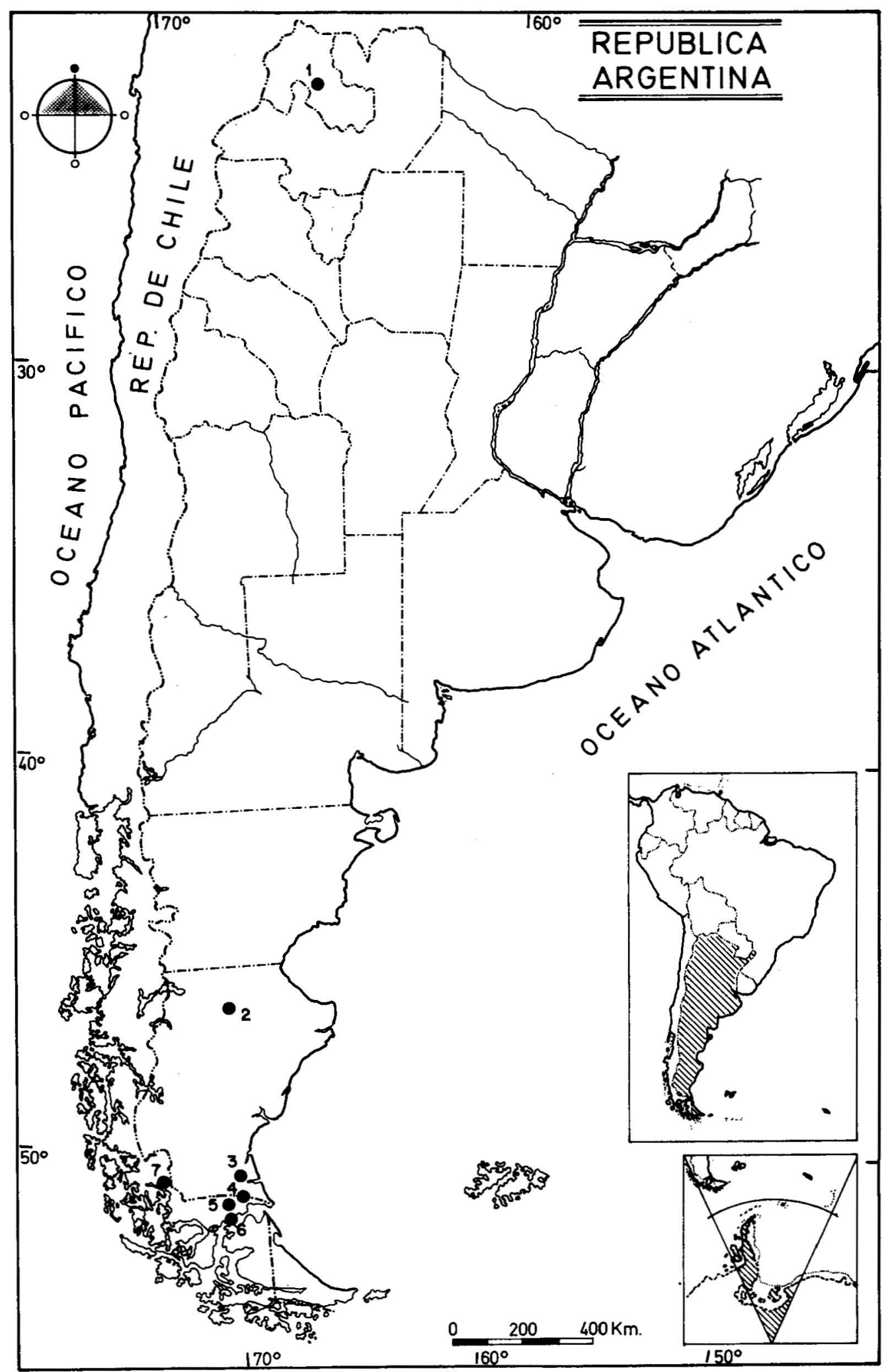

Fig. 1.-Situación geográfica de las distintas localidades: 1. Barro Negro.-2. Los Toldos II y III.-3. Las Buitreras.-4. Pali Aike.-5. Cueva Fell.-6. Cerro Soto-7. Cueva del Mylodon. 
Cuadro 1.-Fauna asociada en los niveles con Hippidion sp.

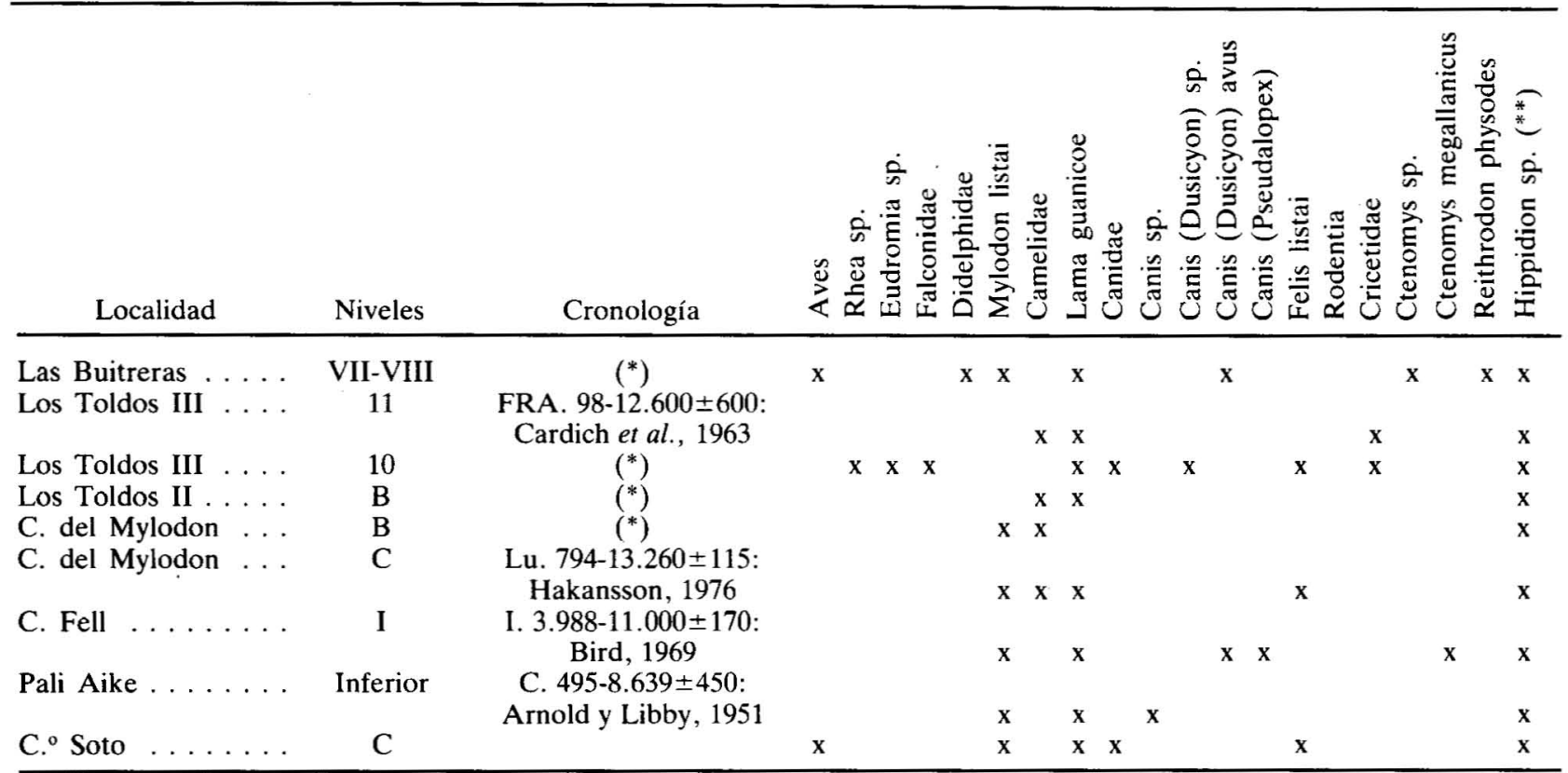

(*) Cronología estimada. Ver texto.

$\left({ }^{* *}\right)$ Denominación taxonómica unificada en este trabajo.

En sus excavaciones estos autores diferenciaron 14 niveles naturales, de los cuales los niveles 10 y 11 contienen restos de caballo.

Estos materiales fueron determinados por Hoffstetter como Onohippidium (Parahipparion) (Cardich et al., 1973).

La cronología de referencia para estos restos se basa en dos fechados obtenidos de muestras de carbón de las capas 9 y 11. Las edades radiocarbónicas resultantes son $8.750 \pm 480$ y $12.600 \pm 600$ (Cardich et al., 1973).

\section{Sitio II de Los Toldos}

Los materiales faunísticos fueron colectados por Menghin en 1951, 1952 y estudiados por Mengoni Goñalons (1980). Este último menciona la presencia de un molariforme inferior derecho (n. ${ }^{\circ}$ 1.460, Museo Etnográfico de Buenos Aires), determinado como Onohippidium (Parahipparion) saldiasi.

El material proviene del nivel inferior B de Menghin, el cual se considera correlacionable con el nivel 10 del sitio III de la misma localidad (Mengoni Goñalons, 1980).

\section{Cueva Las Buitreras}

La cueva Las Buitreras se halla en el curso me- dio del río Gallegos (provincia de Santa Cruz, Argentina). Los resultados de las excavaciones dirigidas por Sanguinetti de Bormida se encuentran en Sanguinetti de Bormida (1976), Sanguinetti de Bormida y Borrero (1977), Caviglia (1976, 1978), Caviglia et al. (1976, 1981, 1986). Estos autores diferenciaron 8 niveles estratigráficos de los cuales los 2 más profundos presentan restos de fauna extinguida.

En el nivel VIII se registran dos P2 superiores, derecho e izquierdo, referidos a Hippidion sp. (Sanguinetti de Bormida y Borrero, 1977), posiblemente pertenecientes a un mismo individuo.

Revisiones posteriores hechas por los mismos autores consideran los niveles VII y VIII como pertenecientes a una misma unidad litoestratigráfica.

No existe datación absoluta para las capas VII y VIII, pero los trabajos antes citados presentan la siguiente aproximación:

1. La base de la capa $V$ fue fechada en $7.670 \pm 70$ BP (CSIC 372).

2. La capa VI de ceniza volcánica corresponde a la erupción I de Fuego Patagonia, fechada para el área en 9.100 BP (Auer, 1974).

3 . Un nivel de bloques desprendidos de la cueva se considera sincrónico de otro similar en la cueva Fell (50 km al SE), fechado entre los 9.030 y 10.600 BP (Saxon, 1976). 
Por lo tanto, las capas con fauna extinguida serían anteriores a estos fechados (Sanguinetti de Bormida y Borrero, 1977).

\section{Cueva Fell}

La cueva Fell se encuentra situada a los $52^{\circ} 4^{\prime}$ $\mathrm{S}$ y $70^{\circ} \mathrm{W}$ en las márgenes del río Chico (Chile). La cueva fue estudiada en un principio por $\mathbf{J}$. Bird, en 1938, quien diferenció 5 niveles.

En el nivel más antiguo (capa I) se hallaron restos óseos de caballo determinado como Onohippidium (Bird, 1938). Estos materiales se encuentran depositados en el American Museum of Natural History de New York.

Posteriormente el sitio fue trabajado por Emperaire, Laming-Emperaire y Reichlen, en 1963. Estos autores reconocieron 14 capas naturales. Restos de caballos fueron recuperados de las capas 9 a la 12. Las capas 9 y 10 corresponden a la transición entre los niveles I y II de Bird, en tanto que las capas 11 y 12 corresponden al I.

Los restos de caballos, estudiados por PoulainJoisien, fueron asignados a Hippidion sp. (Emperaire et al., 1963). Este material se encuentra depositado en el Musee del'Homme, París.

Muestras de carbón coleccionadas por Bird en 1969 , correspondientes al nivel I y a la transición entre éste y el II, proporcionaron respectivamente los siguientes fechados:

I 13988 11.000 \pm 170 , en Bird, 1969: 52.

I-II $1514610.080 \pm 160$ en Bird (com. per. en Ortiz Troncoso, 1981).

\section{Cueva Pali Aike}

La cueva se encuentra situada entre el estrecho de Magallanes y el río Gallegos, a los $52^{\circ} 10^{\prime} \mathrm{S}$ y $69^{\circ} 35^{\circ} \mathrm{W}$.

El sitio fue excavado por Bird en 1936 y 1937 , consecutivamente (Hyslop, en prensa). Diferenció 6 niveles, recuperando de la base de la secuencia restos de caballo americano en las capas inferiores. Estos fueron determinados como Onohippidium.

Posteriormente la datación radiocarbónica efectuada por Arnold y Libby (1951) sobre restos de caballos arrojó un fechado de $8.639 \pm 450$ C486.

\section{Cerro Soto}

Este yacimiento, trabajado por Bird durante la misma campaña (1936-1937), se encuentra situa- do al sur del río Chico, aproximadamente a $1 \mathrm{~km}$ al sur de la Gruta Fell (Chile). De cuyo nivel estratigráfico $\mathrm{C}$ provienen los restos de caballo reseñado.

\section{Paleontología sistemática}

Orden: Perissodactyla Owen, 1898.

Subfamilia: Equinae Steimmann and Döderlein, 1890.

Género: Hippidion Owen, 1869

Hippidion sp.

\section{Descripción del material}

El material de caballo coleccionado por Hauthal en la cueva del Mylodon (1889), revisado en este trabajo es el siguiente: MLP 81-IV-28-4: falange III; MLP 81-IV-28-5: falange II; MLP 81IV-28-6: falange I; MLP 81-IV-28-7: falange I;

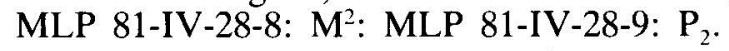

Respecto a los restos recuperados en la expedición del mismo autor en 1900 son: MLP 6-391: porción proximal de cúbito y radio derecho; MLP 6-160: fragmento de rama mandibular derecha con $\mathrm{M}_{3}$; MLP-6-23: fragmento de rama mandibular derecha con $\mathbf{P}_{3}-\mathbf{M}_{2}$ (altura a nivel del $\mathbf{P}_{4}-\mathbf{M}_{1}$, $83,5 \mathrm{~mm}$ ) (ver tabla 2); MLP 6-455 metatarsiano III izquierdo; MLP 6-403: metatarsiano III derecho; MLP 6-456: metacarpiano III con Mc II y Mc IV soldados (ver tabla 1).

Este material es el más representativo de los disponibles para este análisis.

La mandíbula es robusta con una dentición típicamente hipidiforme. Los premolares son marcadamente mayores que los molares.

En cuanto a las características de los metápodos son francamente más cortos y más anchos que los conocidos dentro del grupo. Esta característica propia de este morfotipo los asemeja a las formas terminales descritas para la localidad de Barro Negro (provincia de Jujuy) (Alberdi et al., 1986). Estos ejemplares alcanzan los valores más altos del índice de robustez (tabla 1).

Los metacarpianos II y IV, muy robustos, se conservan soldados al metacarpiano III, sobrepasando la diáfisis de éste (lámina I, figura a).

Las primeras falanges son cortas y anchas, con las tuberosidades distales y el trigonio muy desarrollados como consecuencia de las fuertes inserciones musculares (lámina I, figuras c y d).

El material considerado, de la cueva Las Buitreras consiste en dos premolares $\mathrm{P}^{2}$, derecho e 


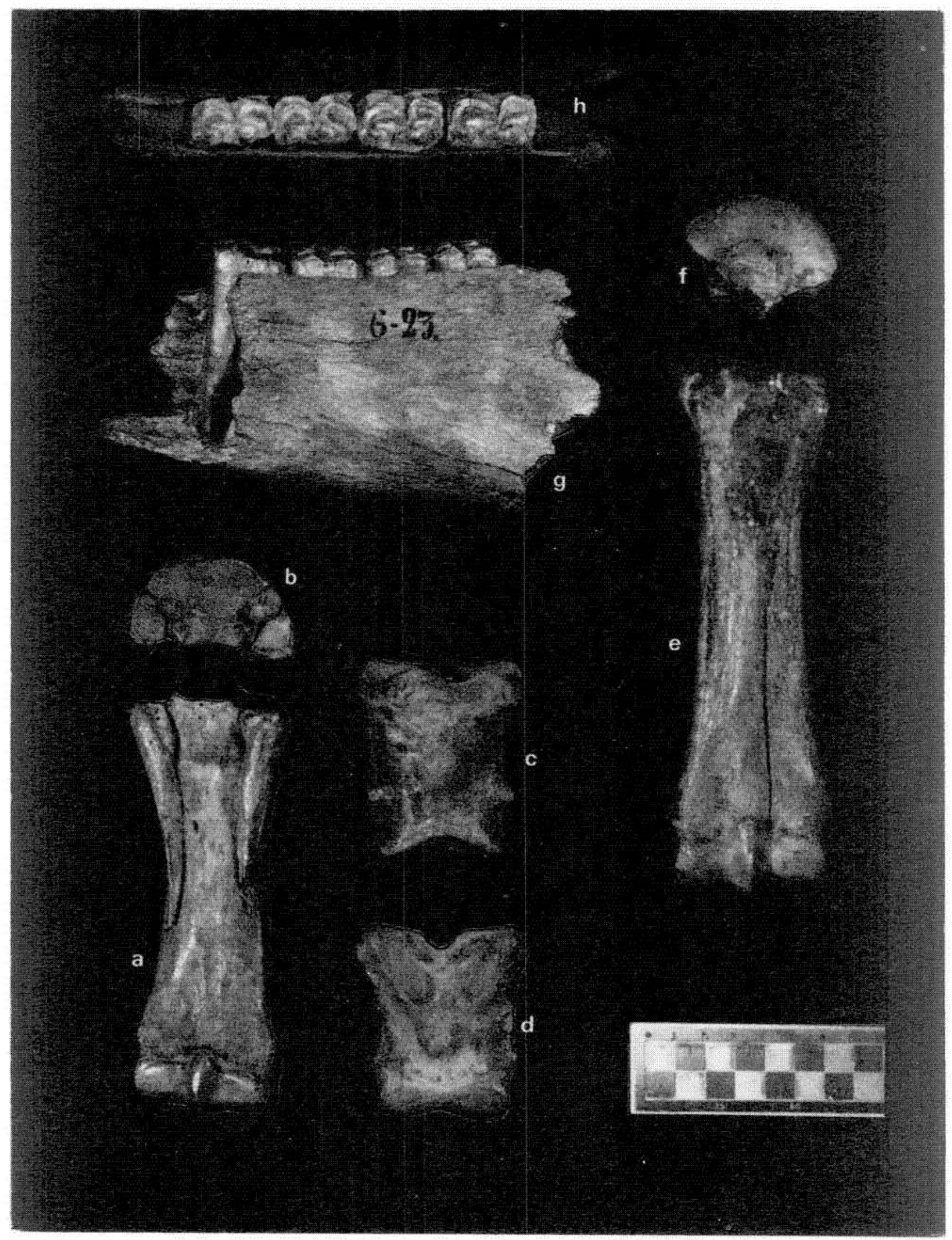

Lámina I.-Materiales fósiles de la Cueva del Mylodon (Chile), depositados en el Museo de La Plata (Argentina). Todos a la misma escala: Metacarpiano III derecho con McIl y McIV soldados (MLP 6-456): a) Vista posterior. b) Articulación proximal-Primerá falange del dedo tercero (MLP 81-VI-28-7): c) Vista anterior, d) Vista posterior.-Metatarsiano III izquierdo (MLP 6-455): e) Vista posterior. f) Articulación proximal. - Fragmento de mandibula izquierda con $\mathrm{P}_{3}-\mathrm{M}_{2}$ ( $\mathrm{MLP}_{6}$-23): g) Vista externa o tabial. h) Vista oclusal. 
Tabla 1.-Dimensiones de los restos procedentes de Cueva Mylodon (Chile) y Los Toldos (Argentina)

\begin{tabular}{|c|c|c|c|c|c|c|c|c|c|c|c|c|c|c|c|c|c|c|}
\hline \multicolumn{19}{|c|}{ Caracteres } \\
\hline & Sigla & 1 & 2 & 3 & 4 & 5 & 6 & 7 & 8 & 9 & 10 & 11 & 12 & 13 & 14 & 15 & 16 & Obs. \\
\hline \multirow{4}{*}{$\begin{array}{l}\text { Metatarsiano III } \ldots \ldots \\
\text { Metacarpiano III ....... }\end{array}$} & MLP 6-455 & 184,7 & 174,4 & 36,5 & 29.3 & 50,1 & 26.2 & 42.8 & 10 & - & 56.5 & - & 34.1 & 24.5 & 28.4 & 105 & - & Izquierdo \\
\hline & MLP 6-403 & - & 171,3 & 35,1 & 28.2 & 50,1 & - & 43,8 & 10.1 & 4.4 & 53.7 & - & - & - & 28,9 & - & - & Derecho \\
\hline & MLP 6-456 & 150,3 & 139,4 & 36,5 & 24 & 44,4 & 31,5 & 37.9 & 11.9 & 5.3 & 52 & 49.4 & 32.9 & 24.6 & 26.3 & 100 & 9.7 & Derecho \\
\hline & & 1 & 2 & 3 & 4 & 5 & 6 & 7 & 8 & 9 & & & & & & & & \\
\hline \multirow{3}{*}{ Falange $I \ldots \ldots \ldots \ldots$} & Los Toldos & 67,2 & 59,1 & 38.6 & 51 & 33 & 43,6 & $\$ 0.6$ & 24.4 & 39.3 & & & & & & & & \\
\hline & MLP 81-VI-28-6 & 64.7 & 56,1 & 39 & 50.5 & - & 47 & +1.6 & 23.2 & 34 & & & & & & & & \\
\hline & MLP 81-VI-28-7 & 68.6 & 56.6 & 45.4 & 59.2 & 36.7 & 50.6 & 44 & 26.7 & 36.9 & & & & & & & & \\
\hline \multirow{2}{*}{ Falange III } & MLP 81-VI-28-4 & 47,2 & - & 73 & 48 & 16 & 37 & 55 & 150 & & & & & & & & & \\
\hline & MLP 81-VI-28-5 & 48 & 52,6 & 61 & 49.2 & 21.5 & 41.3 & 50 & 160 & & & & & & & & & \\
\hline
\end{tabular}

Tabla 2.-Dimensiones del ejemplar MLP 6-23 de la Cueva Mylodon (Chile)

\begin{tabular}{|c|c|c|c|c|}
\hline & \multicolumn{2}{|c|}{ Superficie } & \multicolumn{2}{|c|}{ Base } \\
\hline & Long. & Anch. & Long. & Anch. \\
\hline$P_{3} \ldots \ldots \ldots$ & 27,5 & 16,0 & - & 19,2 \\
\hline $\mathrm{P}_{4} \ldots \ldots \ldots$ & 27,2 & 17,2 & 一 & 19,9 \\
\hline$\vec{M}_{1} \ldots \ldots \ldots$ & 25,5 & 13,5 & - & - \\
\hline $\mathbf{M}_{2} \ldots \ldots \ldots$ & 25,9 & 12,9 & - & 16,3 \\
\hline
\end{tabular}

izquierdo, posiblemente pertenecientes al mismo individuo (Caviglia et al., 1986: 304. Fig. 24).

Este material, por sus características y dimensiones estaría dentro del rango de variabilidad conocido para Hippidion. Sin embargo los dientes yugales de este morfotipo son poco conocidos, por lo cual la contrastación de esta hipótesis depende de la obtención de nuevos materiales.

Los restos disponibles de la cueva III de los Toldos consisten en un premolar superior deciduo, un fragmento de molariforme inferior y una falange I. Esta última presenta las mismas características que el material mencionado previamente para la cueva del Mylodon. Asimismo los parámetros osteométricos (ver tabla 1) concuerdan con los rangos conocidos para este morfotipo, descrito para Barro Negro (Alberdi et al., 1986).

El sitio II de los Toldos suministró dos molariformes, los cuales, por sus descripciones (Mengoni Goñalons, 1980), corresponderían a este morfotipo.

Bird, en sus notas de campo sobre Patagonia (1936-1937), menciona el hallazgo, en la localidad de Cerro Soto, de interesantes restos óseos de caballo y una novedad: restos de pelo (Hyslop, en prensa).

El material en cuestión consiste en: 3 mandíbulas, 1 canino, 2 molares, 1 metatarso, 2 falanges I, 12 falanges II, 3 falanges III y 1 rótula.
Asimismo las campañas realizadas en la cueva Fell tanto por Bird (oo.cc.) como por la Misión Francesa (Laming-Emperaire et al., 1963), dan cuenta de numerosos restos de caballo. Los materiales recuperados por Bird consisten en 3 fragmentos craneales, 2 maxilares, 9 incisivos, 4 caninos, 64 molariformes, 1 atlas, 1 axis, 7 vértebras torácicas, 1 vértebra sacra, 1 vértebra caudal, 1 escápula, 1 húmero, 1 radio, 1 cúbito, 4 metápodos, 1 calcáneo, 1 metatarso, 3 metapodios, 4 falanges I, 3 falanges II y 1 falange III, 3 fémures, 2 tibias y 1 rótula (Hyslop, en prensa).

En tanto que los materiales coleccionados por la Misión Francesa consisten en: 1 fémur, 2 cuneiformes, 4 escafoides, 2 piramidales, 1 trapezoide, 1 cuboide, 1 escápula izquierda, 1 vértebra cervical, 2 incisivos centrales, $1 \mathrm{P}^{3}$ y 3 incisivos deciduos (Poulain-Joinsien, 1963).

Si bien hasta el momento no hemos tenido acceso a estos materiales, la bibliografía original da cuenta de ellos, en cada caso, de la siguiente manera:

«...(these horse)... it was apparent they were of smaller, stokier animals than those used in $\mathrm{Pa}$ tagonia today» (Bird in Hyslop, 1985, Chapter 3), en el primero, $y$

«Est un animal de la taille d'un poney, mais très robuste...» (Poulain-Joisien, 1963: 252), en el segundo.

\section{Discusión y conclusiones}

El material analizado en este sentido ha sido mencionado por los distintos autores bajo denominaciones $\tan$ variadas como: Hippidion, Onohippidion, Onohippidion saldiasi, Hippidium, Parahipparion saldiasi, Onohippidium ( $\mathrm{Pa}$ rahipparion) saldiasi. 
Este es un claro ejemplo del tratamiento sistemático del que ha sido objeto este grupo. Sin embargo, tanto la morfología como las relaciones osteométricas sugieren que estos restos corresponderían a un mismo morfotipo. Por lo tanto hemos convenido en referir todos estos restos a Hippidion $\mathrm{sp}$. hasta tanto no se concluya una revisión sistemática de todas las formas sudamericanas.

Como se desprende, tanto del análisis del material como de la bibliografía consultada, esa forma particular caracterizada por miembros robustos y una talla pequeña, presenta una marcada similitud con la descrita para la localidad de Barro Negro (Alberdi et al., 1986).

Estos hallazgos, de edades radiocarbónicas entre 13.000 y 8.000 BP (Pleistoceno final y Holoceno temprano de Patagonia) y la fauna asociada en cada uno de los niveles considerados hacen suponer una relativa sincronía de estos hallazgos, concordantes, a su vez, con los de Barro Negro y representan la última aparición de formas de este tipo en el registro paleontológico.

El ambiente predominante en la mayoría de las localidades especificadas en este análisis se caracteriza por condiciones rigurosas, ya sea por factores altitudinales o latitudinales involucrados. Esto nos permite hipotetizar acerca de una posible adaptación de este morfotipo a condiciones extremas.

\section{Referencias}

Alberdi, M. T. (1974): El género Hipparion en España. Nuevas formas de Castilla y Andalucía, revisión e historia evolutiva. Trabajos sobre Neógeno-Cuaternario, CSIC, 1, 1-146.

Alberdi, M. T.; Fernández, J.; Menegaz, A. N., y Prado, J. L. 1986: Hippidion sp Owen 1869 (Mammalia, Perissodactyla), en sedimentos del Pleistoceno tardío de la localidad Barro Negro (Jujuy, Argentina). Estudios geol., 42, 487-493.

Aparicio, F. de (1933): Viaje preliminar de exploración en el territorio de Santa Cruz. Publicaciones del Museo Antropológico y Etnográfico, Buenos Aires, 3: 71-92.

Arnold, J. L. y Libby, W. F. (1951): Radiocarbon Dates. Science, 113: 111-120.

Auer, V. (1956): The Pleistocene of Fuego Patagonia. Part. I The Ice and Interglacial Ages. Ann. Acad. Sci. Fennica., 3, 45: $1-226$

— (1970): The Plesitocene of Fuego Patagonia. Part. V. Quaternary Problems of Southern South America/ Ann. Acad. Sci. Fennica, 3, 100: 1-194.

(1974): The Isorhythmicity Subcequent to the Fuego Patagonia and Fenoscandian Ocean Level Transgressions of the Latest Glaciation. Ann. Acad. Sci. Fennica, 3, 115: 1-88.

Bird, J. (1938): Antiquity and migrations of the Early inhabitant of patagonia. Geographical Review, 28: 250-255.

- (1969): A comparison of South Chilenian and Ecuadorian «Fishtail» Projectil Points. The Kroeber Antropological Society Papers, 40:57-71.

Borrero, L. A. (1977): La extinción de la megafauna: su explicación por factores concurrentes. Anales del Inst. de Patagonia, 8:81-93.
Bryan, A. L. Ed. (1986): New evidence for the Pleistocene peopling of the americas. Center for the Study of Early Man. Un. of Maine.

Cardich, A. (1977): Las culturas Pleistocénicas y Postpleistocénicas de Los Toldos y un bosquejo de la prehistoria de Sudamérica. Obra Centenario Mus. La Plata, Antropologia. 1: $1+9$. 172 .

Cardich. A.; Cardich, L., y Hayduk, A. (1973): Secuencia arqueológica y cronología radiocarbónica de la Cueva III de Los Toldos. Relaciones Soc. Arg. Antrop., 12: 85-123.

Cardich, A. y Miotti, L. (1983): Recursos faunísticos en la economía de los cazadores de Los Toldos (Santa Cruz, Argentina). Relaciones Doc. Arg. Antrop. 15: 145-157.

Caviglia. S. E. (1976): Sobre la presencia de un cetáceo en asociación con Hippidium-Onohippidium (sl) y Mylodon en la cueva de Las Buitreras. Relaciones de la Soc. Arg. de antrop.. 10: $313-314$

- (1978): La presencia de Dusicyon avıs (Burmeister), 1864. en la capa VIII de la Cueva Las Buitreras (Patagonia, Argentina), su relación con otros hallazgos de la Patagonia Meridional. VI. Cong. Arqueol. de Uruguay. Salto.

Caviglia. S. E. y Figueredo Torres, M. J. (1976): Material faunístico de la cueva Las Buitreras. Departamento Gure Aike. provincia de Santa Cruz. Relaciones Soc. Arg. Antrop., 10: 315-319.

Caviglia, S. E.; Yacobaccio, H. D., y Borrero, L. A. (1976): Los niveles con megafauna de Las Buitreras: componentes culturales y faunísticos. Comisión XII. X Cong. Un. Inter. Cienc. Prehistóricas, 68-69, México.

(1981): Los niveles con megafauna de Las Buitreras: componentes culturales y faunísticos. $X$ Cong. UISPP. Comisión 12. El poblamiento de América, 68-69.

(1986): Las Buitreras: «Convivencia del hombre con fauna extinta en Patagonia Meridional»: In: New evidence for the Pleistocene Peopling of the America. A. L. Bryan (ed.). Un. of Maine: 295-313.

Eisenmann, V. (1979): Les Chevaux (Equus sensu lato) fossiles et actuels: étude craniologique et odontologique. Tesis doctoral Univ. París, VI: 1-444.

Emparaire, J.; Laming, A., y Reichlen, H. (1963): La Grotte Fell et autres Sites de la Region Volcanique de la Patagonie Chilaine. Jour de la soc. des Americanistes, 2: 168-261.

Feruglio, E. (1950): Descripción Geológica de la Patagonia. I,2 y 3. Ministerio Ind. y Com. de la Nac., Div. Gen. Y.P.F. Buenos Aires.

García Castellanos, T. (1984): Geologia Regional Argentina. Ac. Nac. de Cienc. de Córdoba, 2: 1-1.717.

Hakanson, S. (1976): University of lund radiocarbon dates IX. Radiocarbon, 18: 290-320.

Hauthal, R. (1899): Reseña de los hallazgos en las cavernas de Ultima Esperanza (Patagonia Austral). Rev. Mus. La Plata, 9: $411-420$

Heusser, C. I. (1966): Late Pleistocene polen diagrams from the province of Llanquihue, Southern. Chile. Amer. Phils Soc. Trans., Filadelfia, 4: 269-305.

(1974): Vegetation and climate of the South Chilean District During and Since the Last Interglaciation. Quarternary Research, 4: 290-315.

Hyslop, J.: Travels and Archaeology in South Chile. M. S. Bull. Amer. Mus. Nat. Hist., en prensa.

Lehmann Nistche, R. (1899): Coexistencia del hombre con un gran desdentado y un équido en las Cavernas Patagónicas. Rev. Museo La Plata, 9: 455-473.

(1902): Nuevos objetos de industria humana encontrados en la Caverna Eberhardt en la Ultima Esperanza. Rev. Museo La Plata, 11: 1-16.

Menghin, O. (1952): Fundamentos cronológicos de la Prehistoria de Patagonia. RUNA, 5: 23-43.

(1965): Origen y desarrollo racial de la especie humana. 2." ed. Comp. Nova de iniciación. Buenos Aires, 1-136.

Mengoni Goñalons, G. L. (1976-1980): Los materiales óseos de 
la Cueva II de Los Toldos (expedición Menghin) y una aproximación a la metodología de análisis de restos faunísticos RUNA, 13, 1976-1980: 59-68.

- (1983): Prehistoric utilization of faunal resources arid Argentina (Eds.). Clutton-Brock and Grigson. In: Animals and Archaelogy: I. Hunter and their prey, 325-335.

- (1986): Patagonia Prehistory: Early explotation of faunal re sources (13.500-8.500 BP). A. Bryan (ed.). In: New evidences for the Pleistocene Peopling of the Americas. Center for the Study of Early Man. Univ. of Maine.

Mercer, P. (1968): Glacial variation of some Patagonian glaciers since to Late Glaciar. Am. Jour. Science, 266: 91-109.

_ (1976 a): The Last Glaciation in Chile: A Radiocarbon Dated Chronology. Acta I Cong. Geol. Chileno, Santiago de Chile, 1: D55-D68

(1976 h): Glacial history of southern most South Americal . Quaternary Research, 6: 125-166.

Nordenskjöl, E. $(1900)$ : Jakttagelser och fyndi i grotter vid Ultima Esperanza i Sydvestra Patagonian. Kungl. Svenska Vetenskaps-Akademiens Handlingar, 33: 1-24.

Ortiz Troncoso. O. R. (1981): Inventory of radiocarbon dates from southern Patagonia and Tierra del Fuego. Jour. Soc. des Americanistes. 67: 188-212.

Paskoff. R. P. (1977): Quaternary of Chile: The state of Research. Quaternary Research, 8: 2-31.

Poulain-Josien, T. (1963): La Grotte Fell. In Emperaire et al., 1963. Jour. Soc. des Americanistes, 2: 230-253.
Roth, S. (1899): El mamífero misterioso de la Patagonia «Grypotherium domesticum». II. Descripción de los restos encontrados en la caverna de Ultima Esperanza. Revista del Museo de la Plata, 9: 421-453.

Roth. S. (1902): Nuevos retos de mamíferos de la Cueva Eberhardt en Ultima Esperanza. Rev. Mus. La Plata, 11: 1-21.

Riccardi. A. C. y Rolleri, E. O. (1980): «Cordillera Patagónica Austral», en Gcología Regiona! Argentina. Ac. Nac. Cienc. Córdoba, 2: 1.173-1.306.

Sanguinetti de Bormida. A. C. (1976): Excavaciones prehistóricas cn la Cueva de Las Buitreras (provincia de Santa Cruz). Rel. Soc. Arg. Antrop., 10: 271-292.

Sanguinetti de Bormida, A. C. y Borrero, L. A. (1977): Los niveles con fauna extinta de la Cueva Las Buitreras. Relaciones de la Sociedad Argentina de Antropologia, 11: 167-175.

Saxon, E. C. (1976). La Prehistoria de Fuego-Patagonia: colonización de un hábitat marginal. An. Inst. Pat. Punta Arenas, Chile, 7: 63-74

(1978): Natural Prehistory: Archaelogy and Ecology at the Ultermost Part of the Earth. Monograph Dto. Antrop. Univ. of Durhan.

Tambussi, C. y Tonni, E. P. (1985): Aves del sitio arqueológico Los Toldos, Cañadón de las Cuevas, provincia de Santa Cruz (Rep. Argentina), Ameghiniana, 22: 69-74.

Recibido el 30 de septiembre de 1986 Aceptado el 26 de diciembre de 1986 